\title{
Philosophiques
}

\section{Sans trop mâcher les mots, percevoir \\ Contribution au Réjean Ducharme, Nietzsche et Dionysos de \\ Renée Leduc-Park (Québec, Presses de l’Université Laval, 1982).}

\section{Robert Hébert}

Volume 11, numéro 1, avril 1984

URI : https://id.erudit.org/iderudit/203250ar

DOI : https://doi.org/10.7202/203250ar

Aller au sommaire du numéro

Éditeur(s)

Société de philosophie du Québec

ISSN

0316-2923 (imprimé)

1492-1391 (numérique)

Découvrir la revue

Citer cet article

Hébert, R. (1984). Sans trop mâcher les mots, percevoir / Contribution au Réjean

Ducharme, Nietzsche et Dionysos de Renée Leduc-Park (Québec, Presses de

l’Université Laval, 1982). Philosophiques, 11(1), 191-202.

https://doi.org/10.7202/203250ar d'utilisation que vous pouvez consulter en ligne.

https://apropos.erudit.org/fr/usagers/politique-dutilisation/ 


\title{
ÉTUDE CRITIQUE
}

\section{SANS TROP MÂCHER LES MOTS, PERCEVOIR}

\author{
Contribution au Réjean Ducharme, Nietzsche et Dionysos de Renée Leduc- \\ Park (Québec, Presses de l'Université Laval, 1982).
}

par Robert Hébert

\section{Le titre d'un livre est annoncé par l'éditeur . . .}

Voici donc un livre, Réjean Ducharme, Nietzsche et Dionysos : sur une œuvre constituée de livres dressant la somme des prédicats d'un certain sujet reconnu et masqué, fondateur d'une République Autocratique dont les personnages souvent défenestrés de leur chambre défenestrent à leur tour les nouveaux lecteurs. À partir d'autres livres qui cherchent à nommer le corpus d'un autre sujet dont l'œuvre et les états valétudinaires sont abondamment commentés par des glossateurs-vampires et par des épigones parfois heureux de participer aux institutions impériales de l'Autorité ; à partir de livres qui construisent des catégories englobantes, posthumes, découpant des géographies diverses, des époques avec leurs conditions matérielles précises, locales, monstrueusement données à décoder (Grèce, Orient des sortilèges, Europe, Allemagne prussienne . . .) qu'il n'est pas toujours facile de traduire pour notre présent : comment le dionysisme débarque-t-il au pays ? "Ô jeux de mots ! Ô répandateurs de ténèbres ! Tuez le mot, le funeste, le semeur de confusion. Le 'mot tigre n'est pas un tigre. Qui le sait ? Personne. ". Le mot nihilisme n'est pas une nihiliste ni le vrai rien, Dionysos n'est pas ce verre de vin, le concept de cri n'est pas un cri, mais alors que voulons-nous faire advenir par le cri ? qui n'était pas. Pourquoi pas un Ducharme et la philosophie analytique? Ô|nominalisme des créateurs, qui nous fait mourir au « réel » du langage tout en nous forçant à faire un choix radical parce que complémentaire : ou faire bondir, rugir, lacérer les mots dans l'empire des Lettres, ou inventer, construire, projeter de nouveaux algorithmes réflexifs, du franciscain Guillaume d'Occam à l'américain Nelson Goodman dans l'après coup de sa traduction québécoise.

Voici un beau livre sûrement, parmi les livres d'une collection (parfois désuète) «Vie des lettres » qui a médité pour nous quelques voix caustiques, un peu brûlées par le désarroi d'être ici, historiquement nerveuses et créatrices comme si la ceinture bouclée au dernier trou du langage n'empêchait pas de 
penser cette mobilisation territoriale dont ils étaient eux-mêmes l'instrument ; le Hubert Aquin de Françoise Maccabée Iqbal (1978), le Napoléon Aubin ( "Faut-il donc me casser éternellement la tête à vous expliquer ma tête ? ") de Jean-Paul Tremblay (1969) ou de Marcel-A. Gagnon, Le ciel et l'enfer d'Arthur Buies (1965), ce chevalier errant mort à l'aube du siècle, cinq mois et un jour après Frédéric Nietzsche. Que lisaient-ils ? comment transformaient-ils les ouvrages qui leur tombaient entre les mains selon les arrivages de bateaux ou d'avions (quels Garneau, quels Renaud-Bray, quels Boussac ?) ; pour qui avaient-ils déjà eu l'illusion apollinienne d'écrire, savaient-ils que la signification de leurs textes abandonnés à l'histoire était d'ordre dionysiaque ? De toute évidence, on a toujours écrit, hurlé, émis des signaux, pensé au Québec mais on ne comprend pas toujours dans la grande élite familiale qui doit se tenir les coudes pour ne pas sombrer dans le néant; de toute évidence, les créateurs brouillent les pistes, n'ont pas le temps de faire leur propre théorie littéraire. L'écrivain révolutionnaire et député Louis-Joseph Papineau avait son Lamennais ; avec beaucoup de finesse, le poète Alphonse Piché a parlé de Spinoza du fond de sa roulotte en Haute-Mauricie ; Lucien Francœur, auteur des Rockers sanctifiés (Prix Émile-Nelligan 1983) et « théoricien de l'Amérique ", nous dit que son philosophe préféré est Gilles Deleuze. Anglomanie typiquement française : sous les persiennes de Peirce en Nouvelle-Angleterre ou des producteurs d'Hollywood, le Québec se fait encore doubler. Les philosophes d'ici s'occupent-ils de cela qui reflète si bien leur condition sociale et intellectuelle ? Syndrome de délaissement : les philosophes doivent immédiatement rayer leur passé et leur présent, puisqu'ils découvrent dans leur immédiat une autre contemporanéité ". . . ki leux tombe dêsus l'occiput " (Ducharme, Les enfantômes). Puis " le grosse Lärm cesse " ( $L$ 'biver de force) ; déçus par le résultat de leur enquête ou de leur carrière, le découragement les abat silencieusement. Qui le saura?

Réjean Ducharme aurait donc son Nietzsche murmure une voix, du côté de ce champ (multidisciplinaire) que représentent les sciences de la littérature. Comment et pour qui - au double sens communicateur et aperceptuel de la préposition -, voilà les premières questions de méthode. Mais avant d'ouvrir le livre, relisons par exemple une recension de Raoul Duguay, alors professeur de philosophie au Collège de Maisonneuve et qui n'avait pas encore inversé l'euphonie de son nom propre : « L'avalée des avalés ou l'avaleuse des avaleurs ", Parti pris, IV (1966), no. 3. Sont chantées ici des considérations statistiques sur les 23 personnages du roman, 10 langues y compris le "bérénicien ", au moins 5 départs d'intention, plus de 300 noms d'animaux, de plantes et de végétaux, 12 situations typiquement québécoises, 14 citations de Nelligan . . . et tout à coup : « . . . il y a un volontarisme absolu fondé sur une subjectivité transcendantale (cf. Husserl) et coïncidant avec un rationalisme pur (plus pur que celui de Descartes) et avec un idéalisme plus puissant et plus incarné que ceux de Kant et de Hegel, bref, un volontarisme existentialiste. Voilà ». Voilà, l'époque souhaitait un père littéraire et une axiologie précise. Pourquoi pas donc un Ducharme et Husserl ? Le philosophe allemand et juif (comme le père de Bérénice Einberg) avait éga- 
lement son violon d'Ingres. Ou à partir de cette belle phrase dans le même roman «Voici ce que je suis : un nuage de flèches qui pensent, qui voient qu'elles volent et vers quelles cibles elles volent. Donc je pense .... », le projet fondateur d'un Ducharme et le cogito cartésien. Larvatus prodeo. Puisque nous n'avons qu'un visage non voulu, inévitable, nous pouvons choisir entre trente grimaces à la lumière d'une photographie sereine : rire et penser à être en pensant à rire.

Ducharme/Nietzsche/Dionysos comme un package deal philosophique? Idée stimulante alors que l'on pourrait tout aussi bien croire que la vision ducharmienne construit sa mise en scène dans l'après coup du drame dionysien, dans la dérision de tous les " abîmes existentiels " et des prétendus messages heintenses, sur le mode apollinien de la mémoire et de la protection inconditionnelle de l'enfance, par un joyeux encodage qui n'a pas besoin d'autres médiations qu'une grammaire qui prend acte de ses conditions territoriales. Tout d'abord, l'écrivain qui fait être l'arbitraire de ce qui n'était pas, sa signature, croit-il à toutes ces histoires de revenants?

\section{L'ouvrage est lu. . .}

En rouge, la jaquette donne une première justification inespérée du titre : "Ce rapprochement inattendu entre les deux auteurs prend tout son sens et sa portée heuristique dans. une analyse très serrée et très fine des romans de Ducharme, qui met en évidence une identité thématique et une communauté de pensée entre l'ironie déconstructive du philosophe allemand et la violence teintée d'humour de l'écrivain québécois ". Il semblerait donc que nous nous dirigions vers l'univers chatoyant de la rhétorique des textes et non pas une compréhension des mécanismes créateurs, situés, uniques. Sous la plume de l'A., cette portée heuristique permet une distribution intentionnelle de problématiques multiples et de lectures qui ne semblent pas poser de problèmes ; après avoir conjecturé - au sens fort du terme, juger par conjecture - un rapport entre la pensée de Nietzsche, la théorie de la Relativité et le récent ahurissement des physiciens devant les anneaux de Saturne, l'A. commande ainsi le principe de son enquête : «C'est donc l'ensemble des traits dionysiens communs à Nietzsche et à Ducharme qui détermine une lecture nietzschéenne de l'écriture de ce dernier. Une telle lecture permet d'en dégager une certaine spécificité, d'arriver à une mise en rapport de divers éléments, laquelle effectue un eeffet de différence qu'on pourrait qualifier de nietzschéen en ce sens que s'y élaborent simultanément l'inscription du néant, la mise en œuvre du nihilisme et la manifestation dionysiaque » (9). Bref, cherchons à relaxer la chronicité de l'argument : des traits dionysiens reconstruits à partir de $\mathrm{x}$ éléments (l'éternel retour, "le répétitif », «le labyrinthique ») engage une lecture nietzschéenne de Ducharme, elle-même construite à partir de y éléments ; et celle-ci implique que la moindre différence ducharmienne z sera rapportée à une loi qui n'émane pas de son corpus, cet " effet de différence " qui l'aplatit au cœur même de sa spécificité. 
D'où la division du livre en trois parties - il est à remarquer qu'aucun nom propre et aucun élément d'histoire n'apparaît à la table des matières. "Le néant " $(25-87)$ développe le concept $d u$ néant (il fallait écrire $d e$, pour ne pas sombrer dans un sémantisme qui a toujours tendance à hypostasier du « réel ») selon trois strates signifiantes : le néant explicité selon la récurrence de certains signifiants tels que rien et vide, le néant implicité (sic) et le néant occulté où le « vide masqué par la productivité " du discours ducharmien " se manifeste dans la dissémination des mots savants, des leitmotive et des nombres, et grâce à une certaine (re)présentation de la sexualité ". "Le nihilisme " (89́-118) porte l'analyse sur les institutions humaines archirespectées qui ont subi la corrosion du temps (religion, mariage, formes diverses de l'autorité). "Le dionysisme »(179-292) développe avec brio certains thèmes généraux comme la dureté, le jeu théâtral, la démesure et l'androgynie dans l'œuvre de Ducharme. Enfin, le tout dernier paragraphe amène au jour tout à coup le contexte géolittéraire de l'expression ducharmienne, dite à la fois abâtardie et "pollinisante ". " Au lieu de la défaite et de la défection", l'hétérogénéité de sa langue "s'avérerait une porte d'accès au Nouveau Monde pour les lecteurs du reste de la francophonie » (298). La critique littéraire au Québec ne serait-elle pas toujours dans cette position privilégiée ? : Hermès traduisant à la frontière les bruits intérieurs du pays, dédouanant la langue et la pensée indigènes, porte-parole pour d'autres sur une autre scène homogénéisée.

Suit une courte bibliographie dont la première partie « Textes critiques de l'œuvre romanesque de Réjean Ducharme " regroupe des références toutes québécoises sauf pour Severo Sardui (sic) dont le texte n'a rien à voir avec Ducharme - pourquei n'avoir pas travaillé la réception anglo-canadienne et américaine de ses œuvres? Nietzsche/Dionysos furent-ils, seront-ils des figures évidentes dans l'ironie d'une search for identity aisément repérable ? La dernière partie «Ouvrages théoriques » rassemble 23 noms propres étrangers (la plupart français) et un $24^{\mathrm{e}} \mathrm{du}$ professeur de philosophie $\mathrm{H}$. Weinmann, qui concerne un résumé journalistique sur ... . Edgar Morin. Aucun renvöi sur les principes de la littérature comparée, comparative literature, ou sur cette ruse de l'histoire : le seul livre québécois sur Nietzsche est signé par le vénérable néo-Canadien Charles Murin, Nietzsche-problème. Généalogie d'une pensée, préf. de L.-M. Régis, o.p., Paris-Montréal, Vrin-P.U.M., 1979. Serait-ce là somme toute la présence du regard autocritique et du questionnement philosophique au Québec ? Clivage radical entre l'ici et l'ailleurs en permanence ici. Qu'en est-il du ducharmisme en Europe qui aura servi une politique (plutôt traditionnelle) de l'exotisme?. Qu'en est-il du « nietzschéisme " au Québec depuis la Révolution tranquille où nous avons brûlé les idées d'autrui', sans laisser de traces ni d'expertises sur le sens " réel " de cette combustion? Nous estimons-nous capables de penser cela par nousmêmes ?

L'ouvrage est relu. . . .

L'intérêt général d'un tel projet, nous semble-t-il, est de raffiner les 
méthodes qui peuvent, avec souplesse, décider, dévoiler ou même engendrer des contextes traducteurs où se pense "l'identité thématique » entre le philosophe allemand et son envers Ducharme ; où se formule également cette "communauté de pensée " qui leur est intuitivement allouée. Nous ferons trois remarques d'ordre méthodologique sur les limites de ce projet, plus précisément sur la rare ambition qui nous est donnée ici à comprendre.

Première remarque. Tous ceux et celles qui ont «potaché " dans Freud, Lacan, Saussure, Benveniste, Bataille, tomberont d'accord sans peine aucune, lexicalement parlant, sur les généreux commentaires de l'A. qui pourtant mettent en abîme l'œuvre de l'écrivain pour la faire surgir, magiquement parlant sous des explications étrangères à sa loi. Lexiques institués, en rien dionysiaques. Ce qui n'est pas sans poser de sérieux problèmes quant aux fondements de l'activité critique et d'une littérature comparée qui prétend avoir des objets. Ces malaises s'entendent par la naïveté hautement sophistiquée du langage et par certaines incongruités qui montrent l'éloignement des débats historico-philosophiques sur Nietzsche et la clôture des disciplines (via la filière française) qu'elle n'interroge jamais. Comment peut-on écrire en 1982 " les spécialistes nietzschéens » (6), " tous les experts nietzschéens s'entendent pour ... (9): entendre quelques-uns, typiques, Pautrat, Lacoue-Labarthe, les créateurs Blanchot, Deleuze et Klossowski, KremerMarietti sur la question européenne du nihilisme . . Q Quant aux « classicistes"(182), deux seules références aujourd'hui mieux situées : Otto en 1933 (profondément romantique et deutschfreundlich), Jeanmaire en 1951, réimprimé en 1970 après les événements de Mai-68. Une nouvelle génération d'hellénistes travaillent cependant d'autres figures. Par exemple une lecture de Dionysos mis à mort de Marcel Detienne (Paris, Gallimard, 1977) aurait pu donner des idées hors du commun : l'interprétation politique du dionysisme par le biais de l'opposition ville/campagne, civilisation/sauvagerie, le thème de la poursuite, de la chasse et de la mise à mort, le ménadisme des personnages féminins, la signification des rituels omophagiques (maculage de sang, lacération des chairs, mastication . . .) et de la cuisine cannibale (" qu'est-ce que ronger la tête de ses parents"?). Les deux derniers sujets sont à peine effleurés par l'A. et c'est dommage. Pourquoi pas un Système culinaire de l'écriture selon Ducharme ? en cent pages, plaquette précise, originale et originaire du corpus ducharmien.

Disséminées à travers le livre, le réseau des citations fonctionne comme une mise en conserve qui a toujours tendance à exhiber une étiquette étrangère à son contenu : " . . . exposer l'activité du texte en tant que producteur d'un sens : l'apparat ducharmien, simulation de l'appareil nietzschéen » (10), présupposé qui va de soi et combat d'arrière-garde d'un côté, twistesse de la connection historique de l'autre côté ; "l'œuvre romanesque de Ducharme réalise donc le paradoxe que Genette décrit . . . " (19) ; " l'assertion apparemment paradoxale que la passivité sexuelle correspond chez Ducharme à une affirmation s'appuie sur les commentaires de Blanchot cités par Bataille dans son ouvrage sur l'érotisme, où il est question de Sade . . . " (83), l'A. 
ajoute même que les remarques de Blanchot " corroborent un autre aspect de la doctrine nietzschéenne "- doctrine pour qui ? ; chez Ducharme, la prolificité de l'écriture génère le texte scriptible selon la signification que, dans $S / Z$, Barthes attribue à ce terme en s'inspirant d'ailleurs de Nietzche . . ." (295). Et partout le clivage entre ce que nous appelerions les embrayeurs coloniaux comme "selon ", " pour ", " d'après " et l'embrayeur domestique par excellence «chez ». Quand pourrons-nous inverser cette loi ? Écrire par exemple : "Selon Réjean Ducharme, le concept de la lacune originelle chez Jacques Lacan . . . » et commencer à méditer avec une information précise les mutations syntactico-sémantiques et le contexte politico-institutionnel du néo-freudisme en France ? Ce qu'ont déjà entrepris les Américains (cf. le livre de Sherry Turkle, Psychoanalytic Politics. Freud's French Revolution, New York, Basic Books, 1978).

Deuxième remarque. L'A. semble vouloir forcer le signataire de L'biver de force à entrer dans une nouvelle camisole de force théorique, traditionnelle ; et c'est la seule limite de l'ouvrage. On voit donc l'ambition du projet qui fait d'une cuvre littéraire le support de certaines idéologies antérieures, de grilles d'analyse historiquement situées. Partout la main qui écrit, ni celle de Nietzsche ni celle de Ducharme, veut prouver ce qu'elle inscrit et non pas ce qu'il signale, attester, démontrer, corroborer, référer avec beaucoup d'autorité à d'autres autorités qui se sont permises de théoriser d'autres contenus, d'affirmer des thèses, de déterminer, de sanctionner ce qu'elle perçoit être leurs acquis. Banalisant par conséquent une tendance scientifique nécessaire au Québec, par où la qubébétude du vrai vrai passe par le fantasme du langage judiciaire, sans objet et sans litige, aliéné à des procès-verbaux qui sont clos. Qu'est-ce que prouver scientifiquement aujourd'hui ? voilà la question ; qu'est-ce que prouver littérairement que l'on prouve ? voilà une autre question ; ou qu'est-ce que ne plus vouloir prouver parce que l'on a éprouvé le problème de l'interprétation et alors qu'est-ce qu'engendrer des concepts nouveaux qui réfléchissent le "réel " de la question ? L'A. ne semble pas s'apercevoir qu'elle construit hors de son triple objet - ce qui n'est pas, bien sûr, erroné car les savoirs disciplinaires et la science ne peuvent que construire ; mais elle prend pour du réel l'arbitraire d'une construction qui d'office élimine le réel autonome de l'œuvre ducharmienne. Habité par une écriture homonymique qui laisse toujours entrevoir l'humanité de ses procédures les plus rigoureuses, fasciné par ce coup de force opéré dans le Vieux Monde du $\mathrm{XIX}^{\mathrm{e}}$ siècle lui-même subjugué par les mille facettes de l'Idéal grec (de Hegel à Marx en passant par Shelley, Pater, les philologues allemands ou Fustel de Coulange), la fatigue alors s'empare du lecteur qui préfere peut-être relire Réjean Ducharme en écoutant un Offenbach rockeur ou les chansons du premier Charlebois. Mais attention, sa marge de manœuvre est presque nulle, toute conscience de l'artefact textuel est annulée, anyway : "De toute façon, si la réception artistique du texte ducharmien en contredit l'interprétation selon une orientation nietzschéenne, cela demeure tout à fait normal, puisque la contradiction est le propre de Dionysos » (31). D'où vient ce puisque ? Après les paradoxes de Grelling, d'Épiménide le crétois et du Barbier, voici l'in- 
jonction paradoxale de Dionysos, ou mieux le paradoxe herméneutique de l'Ennéoué (demeurons fidèle à Ducharme). Ou bien tout lecteur confirme l'orientation nietzschéenne de cette interprétation, auquel cas il confirme le dionysisme de l'œuvre première ; ou bien tout lecteur contredit cette orientation nietzschéenne, auquel cas il manifeste et confirme un esprit dionysiaque digne du nietzschéisme qui englobe l'œuvre première. Pauvre Ducharme ! Logiciens imberbes, au travail, l'avenir vous appartient. Par quel rasoir phosphorescent trancher le rapport au vrai ? et comment résoudre le paradoxe herméneutique de l'Ennéoué ? Voici une solution : l'A. $\alpha$ de la présente recension décide maintenant de contredire, non pas l'orientation nietzschéenne en soi d'une interprétation parmi d'autres, mais bien l'interprétant A. $\beta$, dans son rapport second et « jaloux " à une œuvre collectivement projetée en un point fictif, Oméga.

Troisième remarque. Revenons au tout premier paragraphe du livre qui résume avec la page 297 les précautions de l'A., par la suite occultées dans la pratique même de sa lecture. Incipit : "Réjean Ducharme est au courant des thèses des Nietzsche : deux de ses romans, le Nez qui voque et La fille de Christophe Colomb, en attestent » (5). A-t-il vraiment lu tout le philosophe allemand? Question dépourvue d'intérêt, l'A. en convient. Nous n'avons pas encore l'inventaire public des 69 livres laissés à André Sylvestre à l'été 1965 - cf. J. Blouin et J.-P. Myette, "À la recherche de Réjean Ducharme ", L'actualité, VII (juillet 1982), no. 7, pp. 48-49. Et d'autres philosophes grimacent dans cette œuvre, accollés à des interjections « stupéfiantes " Oh !, Ah !. Comment a-t-il lu le philosophe, s'il l'a lu parmi d'autres philosophes? voilà la question. Pourquoi pas un Ducharme et l'ironie kierkegardienne ou, avec une méthodologie appropriée, une enquête sur le très " ducharmant "Schopenhauer que Nietsche a dû renier au jour, pour advenir à sa propre vérité contre la négation (textuelle) du vouloir vivre. La fin du paragraphe nous rappelle que ces deux " évocations fugaces et fautives sinon truquées des postulats de Nietzsche " ont pour l'A. " déclenché une lecture nietzschéenne, bien que, sur certains points, nous reconnaissions l'incompatibilité de Nietzsche et de Ducharme ". D'où origine cette pensée du compatible ? Pourquoi ne pas avoir fait (pour le bénéfice du lecteur et des philosophes) une théorie de cette incompatibilité, et avoir approfondi la question de "la femme " et de « la mort de Dieu " (entre guillemets) qu'elle expédie d'une manière étonnamment superficielle? Que s'est-il passé dans son programme de recherche entre cet aveu et le paradoxe herméneutique de l'Ennéoué à la page 31 ?

\section{Un dossier "Réjean Ducharme " est consulté . . .}

Reste que tout l'armement conceptuel et la complexité méthodique hors méthodologie produisent du sens, le signe d'une présence intellectuelle et d'un tempérament. Un auteur apparaît, une signature se manifeste et nous en sommes heureux. Le patient travail des références en dit plus que la brève notice biographique du Robert des Noms Propres qui fait naître Ducharme en 1942, alors qu'il est né d'Omer D. et de Nina Lavallée le 13 août 1941 : 
" . . c courts tableaux entrecoupés de cris de révolte ou de réflexions morales, voire de calembours . . . discours étincelant de virtuosité où apparaissent en filigrane les problèmes linguistiques et culturels du Canada français » (1974, p. 562) - analogiquement sans doute, les problèmes linguistiques et culturels de la critique littéraire du Québec. Il n'est pas sûr cependant qu'en voulant prouver à tout prix (à partir de ses références françaises) et en investissant ses énergies à construire comme si elle ne construisait pas, elle ne traduise pas autrement le court jugement que Paule Leduc portait il y a quinze ans sur L'Océantbume - sic, l'orthographie ne présumant pas d'une coquille puisque le titre revient ainsi 7 fois dans la recension de cette dernière : " Le rythme est moins rapide, moins fulgurant que dans L'avalée des avalés. Peut-être y voit-on mieux l'expérience de la fête, de la levée des lois qui conduit à l'inversion des règles morales et à l'inversion du langage ", Études littéraires, II (août 1969), no. 2, p. 206. Interprétation alors bataillienne qu'elle répétait en général pour les romans de Marie-Claire Blais (alors exilée en NouvelleAngleterre), Jacques Ferron (qui ne s'était pas encore enorgueilli de n'être jamais sorti du patelin) et Hubert Aquin qui n'avait pas encore rempli par la mort le trou de sa mémoire. 1968 était une année faste : le Québec découvrait la possibilité du « délire verbal » en littérature.

D'ailleurs, il semblerait que le flou, le vague, l'exotisme chatoyant des attributs et des prédicats qui convergent vers le personnage Ducharme soient à la hauteur de cette imprécision générale : inatteignable comme toute création qui fait être sa loi avant de permettre sa réception, question de méthodologie. Reprendre ici tout le dossier de presse "Réjean Ducharme " serait fastidieux et l'espace manque. Mais dans le domaine français, qui se rappellera «Zazie, du Québec » de Maurice Nadeau (Quinzaine littéraire, 13, 1966) avant la mise en marche du métro de Montréal ; "Le Canadien à l'âge de Rimbaud " d'Étienne Lalou (L'express, 831, 1967). Plus important, "La tactique de la guerre apache appliquée à la littérature " de Le Clézio dont la référence exacte est rarement donnée au Québec (Le Monde, no 7458, 4 janvier 1969, p. VIII), où son portrait de Ducharme se présente comme « un autoportrait " en Peau-Rouge, texte fraternel et narcissique dont l'incipit anti-dionysiaque ( "Les révoltes sont inutiles. Elles n'arrivent jamais jusqu'au bout ") nous relaie à la page 22 de L'Océantume : "Dans le genre révolté, depuis que Aristote s'est masturbé sur la place publique, il n'y a plus rien de surprenant en diable à faire ». Aristote ou Diogène le Cynique selon nos doxographies ? Errance créatrice de l'ironie. Ou encore, "Les blancs d'Amérique » de Claude Roy (Nouvel Observateur, 264, 1969) qui s'appropriait alors un spectre typiquement québécois. Serions-nous des spécialistes de cette twistesse spéculaire dont la glose critique du calembour allègue que " ce processus ludique extirpe du signifiant originel twistesse son signifié de 'morosité, en le déformant. Cette déformation produit une rémanence plus <joyeuse`, celle de la danse, tout en désignant l'activité de l'énonciation présente, celle de «tordre> les mots" (22). Et pour le lexique des futures générations d'auteure-s : celle de « se faire tordre le bras » à chaque poignée de mains territoriale.

Débordement d'hypothèses et d'anecdotes inter-nationales, paradoxe biographique et herméneutique de l'Ennéoué dont on sait qu'il est constitutif 
du Mythe Ducharme dès le début, plus précisément par et grâce à ce début Il y aurait beaucoup à méditer, à théoriser sur la réception de l'œuvre ducharmienne : car vingt ans plus tard, ne serions-nous pas encore dans le fantasme d'une authentification intellectuelle au second degré ? Au premier degré, qui se rappellera le jugement du jeune philosophe Clément Rosset, assistantprofesseur à l'Université de Montréal et alors en mission : "La richesse et la perfection de la langue de l'Avalée des avalés ne posent aucun problème d'authenticité, compte tenu de la culture, de l'intelligence et de la mémoire de Réjean Ducharme. Les divers bruits qui ont couru sur son compte proviennent, semble-t-il, exclusivement de certains milieux littéraires et journalistiques de Montréal que la retraite volontaire de Ducharme indispose. Ces bruits sont dénués de tout fondement ", "Réjean Ducharme authentifié " (et non pas "identifié » comme l'indique la très sérieuse French Bibliography du French Institute de New York), Le Monde, 27 janvier 1967, p. 17. À côté d'un entrefilet sur le méconnu Max Jacob. Comme le dit à tout propos le narrateur de L'Hiver de force, "fuck". Observons que le mot "fuck" n'étant pas l'acte parce qu'il est autre chose dans le texte, il n'est pas seulement un simple explétif qui finit par "structurer l'absence " et " simuler une lacune de vocabulaire " mais aussi (cela, il faut le penser . . .) le sème fondamental d'un écrivain nord-américain qui légitime ainsi les 283 pages d'un récit qui a un commencement parce qu'il a une fin. Ô nominalisme rageur qui démembre sans cesse le corps de l'Humanité pour nous le donner à lire.

\section{Pause. . .}

Qu'est-ce donc qu'un auteur ? Quelqu'un déjà là, sur qui une autre conscience glisse et dérape en connaissance de cause. Et un ensemble d'auteurs ? "Idem au cube ". Mais que faire alors ? Choisir les seuls extrêmes possibles : ou agir et créer par impulsion en tant que corps explorant une autre vie, une autre époque théorétique ; ou réfléchir les conditions d'émergence d'une œuvre et à la limite, faire œuvre de géographe, de politicologue, d'économiste de sociologue dans le champ de l'histoire littéraire. En définitive, une question se pose - le sens de l'expérience humaine à travers l'écriture - parce que tout repose sur l'acte symbolico-juridique qui arrache une persona " au néant " collectivisé et transfigure l'arbitraire de ses déterminants sociaux. Entouré de ses personnages (Bérénice et la famille Einberg, Gloria, Chamomor, Iode Ssouvie, Mille Milles, La Toune, Laïnou, Fériée, Urseule, Sharon . . .), dans l'essoufflement progressif des multiples dédoublements d'André Ferron et de Vincent Falardeau, cet auteur narrateur et ses personnages n'existaient pas avant le 7 septembre 1966 - date de l'achevé d'imprimer pour tout lecteur universel, ce qui correspondait à la rentrée scolaire et académique ici. En langage bérénicien, « Le nom propre est l'origine ; et le seul (néant) qui existe est le néant signalé par chaque signature, à partir de quoi d'autres lecteurs inferent d'autres «néants〉, dans le miroir simulateur de tous les lexiques disciplinaires instituant le nomenclature des 〈néants〉, eux-mêmes institués, morts. Les Profondeurs n'existent pas ; depuis cinq siècles, l'Homme vit dans la méconnaissance de sa Platitude typogra- 
phique. Le mot profondeur n'est pas profond. Qui le sait ? L'enfance. Je suis la mémoire agissante du passé-présent et je vous récite plein d'histoires ». Exeunt par cette existence, le discours adulte et les turlutes manquo-métriques de la psychanalyse, ou son double contraire, le discours adulte et musclé des sciences behaviorales.

Qu'est-ce alors qu'un non auteur ? Un jeune homme revient de Key West avec un coup d'humidité dans les gencives, pour raconter, trouver une solution à cette contradiction que la vie se maintient arbitrairement contre l'impossible vie de l'orgiasme, écrire, s'organiser dans l'irréalité d'une signature qui dès lors où elle se réalise, dissimule parce qu'elle transfigure autant le passé qu'elle anéantit, autant les futures explications qui l'anéantiront. Chaque signature est une solution momentanée à quelques contradictions précises, locales, incorporées : d'où les mises en scène démultipliées de la narration, avec sa syntaxe "coloriée ", son ronron rythmique et typographique toujours plein ... Peut-être a-t-il entendu, "ce jeune homme édenté ", les dernières mélopées dionysiaques des Cajuns : il en revient comme il doit nécessairement revenir de tout. Il doit trouver une solution. Comme Euripide ? comparaison compare ! Euripide déjà "fucké " par les dieux de son Olympe et la Cité grecque souffrait (pour notre bonheur) de ne plus pouvoir écrire des tragédies comme Eschyle. Antériorité du tragique. Sa solution ? Écrire les Bacchantes dont les ambivalences sociales et politiques ahurissent encore les scholars. Mise en scène d'une mise en scène pensée, présidentielle : d'un Maître de cérémonie très apollinien, à heure fixe : "Citoyens et citoyennes, vivons notre pathos en mangeant du pop corn . . . face à face avec celui que vous attendez tous. Quel enthousiasme ! Voici, enfin Dionysos en personne $\rightarrow$ (traduction bérénicienne-de la-catharsis d'Aristote).

Peut-être Réjean Ducharme a-t-il entendu les thèses aux accents gutturaux de Nietzsche qui lui-même a survécu à la pensée de l'éternel retour, pour nous le dire ; raillant sans pitié le pathos, "la démesure irréfléchie ", les excès sentimentaux du romantisme, pour trouver une solution à la fín du $\mathrm{XIX}^{\mathrm{e}}$ siècle, la sienne, celle du stylet et du style. Mais que faire de ses derniers mots, "Je suis Dionysos, le Crucifié "? Relisons la dernière lettre de Turin à Pietro Gast, tampon postal du 4 janvier 1889 : "Chante-moi un chant nouveau : le monde est transfiguré et tous les cieux exultent ». Sérénité accomplie ou dernière extravagance, Narrbeit. Ajoutons en langage bérénicien : " . . . encore fallait-il qu'il colle ou fasse coller le timbre de ses lettres, de ses cartes postales. Le vrai fou aurait avalé le timbre, ou mangé la pinède de la carte postale ". Insignifiance universelle de l'événement (nous souffrons tous, épinglés, endoloris - Dionysos et Christ ne sont que des noms pour le dire), souveraineté apollinienne du geste. Nommons Apollon (dont les attributs sont la lyre et des flèches souvent colériques) Dieu nouveau des Postes, Dieu protecteur des Bibliothèques, des Centres de documentation et des Banques de données. Pour chaque lendemain, le tragique est une question de rétrospective. Relisons maintenant Ducharme, Nietzsche et Euripide dans le texte, leurs langues uniques. 


\section{Le livre est conservé comme un document etbnophilosophique . . .}

Si nous avons pris le temps de dévorer ce discours critique, second, et la talentueuse opération de remythologicisation de l'écriture effectuée par Réjean Ducharme, Nietzsche et Dionysos, c'est que cet ouvrage est important. Il arrive à point. D'une part, il constitue un premier livre sur l'écrivain, dont il faudra tenir compte, une première interprétation radicale de Ducharme, entreprise avec cette ardente sympathie qui travaille à universaliser, à traduire autrement ce qui est déjà universel. D'autre part, il représente un point culminant dans la manière québécoise de mettre en rapports littérature, philosophie et "sciences du Texte ». Par des biais insoupçonnés de son A., il amène à sa limite, notre limite ; il exprime des problèmes épistémologiques de fond pour toute réflexion philosophique sur la notion d'œuvre. Qu'estce qu'un texte littéraire, philosophique, religieux ? Qu'est-ce que comparer d'une manière et pas d'une autre, construire à partir de grilles d'analyse ellesmêmes situées, historiques ? emprunts culturels qui sont rarement médités à partir de leurs points cardinaux, de leurs banques d'emprunts. Qu'est-ce ne pas méditer le statut ontologique de l'être et du non être, du rien, du vide, et hypostasier tout de go la profonde surface de l'écriture ? Nous attendons avec hâte le prochain ouvrage de L'A. : sur sa méthodologie ou Jean Ricardou ou d'autres facettes de Ducharme ? Espérons qu'elle puisse mettre en abîme son propre apparat critique, question de libérer sa propre passion de lire. Pourquoi ne pas vagabonder dans l'histoire rafraîchissante du literary criticism américain depuis 1945 ? où le corpus amène avec lui ses catégories d'analyse territoriale? Dans un esprit ducharmien, pourquoi pas une Théorie de la pluvitude analytique, à partir de l'anagramme "plus tu vides" soigneusement décrypté à la page 35 ? Plus l'analyse coule, plus elle rate et manque son objet. Plus elle doit remplir son vide, plus elle devient excessive, auto-référée, contre son objet.

Dans la mesure où d'autres questions préalables, d'autres travaux fondateurs ne sont pas encore posés par les professeurs de littérature et par les philosophes d'ici - qui perdent avec le temps la « Faculté de ruminer dès l'aurore " - , ce livre pointe du doigt un manque dont il ne saurait être responsable. De son ailleurs, il reflète notre manière irresponsable de faire ou ne pas faire l'histoire de nos idées : là réside aussi son extrême importance. Qu'est-ce que lire Nietzsche et Ducharme depuis la Révolution dite tranquille ? Mainmise revendiquait aussi un Dionysos psychédélique étendu entre quelques champignons bleutés du Mexique. Quelles sont les traces à penser, à remâcher ? Nos universitaires lisent-ils Ducharme et Nietzsche avec cette sorte de complicité ethnophilosophique qui seule peut universaliser notre corpus ? Y a-t-il des experts-philosophes (« nietzschéens » ou autres, cela fait résonner la même question) qui soient vraiment habités par la passion d'un creative scholarship, avec référents textuels à l'appui ? Qu'est-ce que ne pas pouvoir publier toutes les thèses de maîtrise et de doctorat sur Nietzsche, la contre-culture ou Roland Barthes, entre 1968 et 1978 ? Si nous voulons nous soumettre à l'injonction paradoxale de Dionysos, où sont les textes contradicteurs, " contributifs » d'une lucidité minimale, retravaillables pour 
d'autres responsabilités ? Manque de documentation, manque de réflexion dénotée, manque de créateurs dans l'ordre de la pensée. Voilà au Québec, nous brûlons d'être immédiatement dans le bon paradigme. Cash and burn. Vertiges de la vitesse. Les générations se suivent hors de leur historicité clivée par les élites et nous nous lamentons de " ne pas avoir de tradition, comme . . . ». Mais comme toujours, face au hibou crépusculaire de la tradition des virages citationnels, les philosophes se font doubler de tous les côtés, par tous les corpus ; puis « le grosse Lärm cesse ».

Nous rappelant une belle phrase de Ducharme sur le destin : «On est calorifere. On ne peut rien y changer. Les êtres humains sont les seuls caloriferes pouvant ruer dans leur brancard, leur forme ", comment alors ne pas traduire autrement la situation littéraire, philosophique et scientifique du Québec (car un jour on s'apercevra que chacune n'allait pas sans les deux autres) : éternellement, la chair de ses penseurs dit dionysiaques bouilllie dans la tuyauterie institionnelle d'un Apollon de surface, shining. Si nous décidons de prendre ce langage, trouvons une forme appropriée à ce qu'il signale et signifie, et racontons maintenant l'origine des mécanismes créateurs de toute écriture et de toute pensée. Patience dans l'azur. Sous le ciel des structures anamnésiques (Ô Mnémosyne), le retour mythique des Dieux (comme ce Néos Dionysos), des Idoles (comme Nietzsche) et des Génies littéraires (comme Ducharme) s'effectue à chaque fois non pas pour que nous puissions sanctionner et contempler le système absent de leurs idées, mais bien pour que nous les tuions - et ils le savent - au moment même où nous comprenons comment ils ont fabriqué le corps de leurs idées, l'être de leur apparence au seuil de la caverne, du poste douanier ou de la salle de travail, œuvre stimulante parce que dissimulée contre sa propre force, nous forçant à recréer d'autres intensités.

Ingenii alea jacta est : l'ingénierie intellectuelle doit pouvoir s'ouvrir à ce qu'elle recèle de plus puissant dans le tissu conjonctif de son corpus, en rendant nécessaires à chaque fois les lois réfléchies et réfléchissantes de ses aléas, l'aléatoire démographique, politico-social, géographique, par exemple. L'avenir appartient à l'ingénieuse destinée de ceux et de celles qui transforment leurs sources, transforment les solutions habituelles à leurs contradictions précises. À moins que la contradiction impensée ne soit aussi une habitude. Quant à l'auteur de la présente étude, il n'a voulu qu'actualiser le sens de l'événement Ducharme, l'illustrer, par conséquent commémorer un événement qui fait encore sens. Avec les limites de sa mesure et la démesure d'une topique interdisciplinaire. Il espère que Renée Leduc-Park aura perçu que c'était lui rendre hommage que d'écrire tout ceci ; il lui doit d'avoir cheminé avec elle sur les sentiers obliques qu'elle a eu le courage de dessiner. D’autant plus qu'elle a aussi dédié son livre : "pour Réjean Ducharme, le méconnu ».

Beaux souvenirs, silence, hospitalité d'une vie quotidienne qui nous rend tous et toutes à la difficulté de faire être, dans et par l'écriture.

Département de philosophie

Collège Maisonneuve 